\title{
Genotoxic Bystander Signals from Irradiated Human Mesenchymal Stromal Cells Mainly Localize in the 10-100 kDa Fraction of Conditioned Medium
}

\author{
Vanessa Kohl 1, Alice Fabarius 1, Oliver Drews 2, Miriam Bierbaum ${ }^{3}$, Ahmed Jawhar ${ }^{4}$ \\ Ali Darwich ", Christel Weiss ${ }^{5}$, Johanna, Flach 1, Susanne Brendel 1, Helga Kleiner 1, \\ Wolfgang Seifarth ${ }^{1}$, Wolf-Karsten Hofmann ${ }^{1}$ and Henning D. Popp ${ }^{1, *}$
}

1 Department of Hematology and Oncology, Medical Faculty Mannheim, Heidelberg University, 68167 Mannheim, Germany; Vanessa.Kohl@medma.uni-heidelberg.de (V.K.); Alice.Fabarius@medma.uniheidelberg.de (A.F.); Johanna.Flach@medma.uni-heidelberg.de (J.F.); Susanne.Brendel@medma.uniheidelberg.de (S.B.); Helga.Kleiner@medma.uni-heidelberg.de (H.K.); Wolfgang.Seifarth@medma.uniheidelberg.de (W.S.); w.k.hofmann@medma.uni-heidelberg.de (W.-K.H.)

2 Department of Clinical Chemistry, University Medical Center Mannheim, 68167 Mannheim, Germany; Oliver.Drews@umm.de

3 Department of Radiation Oncology, Medical Faculty Mannheim, Heidelberg University, 68167 Mannheim, Germany; Miriam.Bierbaum@medma.uni-heidelberg.de

4 Department of Orthopedics and Trauma Surgery, Medical Faculty Mannheim, Heidelberg University, 68167 Mannheim, Germany; Ahmed.Jawhar@medma.uni-heidelberg.de (A.J.); alidarwich@mail.com (A.D.)

5 Department of Medical Statistics and Biomathematics, Medical Faculty Mannheim, Heidelberg University, 68167 Mannheim, Germany; Christel.Weiss@medma.uni-heidelberg.de

* Correspondence:Henning.Popp@medma.uni-heidelberg.de; Tel.: +49-621-383-71307

† Presented at the Cell-to-Cell Metabolic Cross-Talk in Physiology and Pathology, 17 December 2020 to 17 January 2021; Available online: https://cells2020.sciforum.net/.

Published: date

\begin{abstract}
Genotoxic bystander signals released from irradiated human mesenchymal stromal cells (MSC) may induce radiation-induced bystander effects (RIBE) in human hematopoietic stem and progenitor cells (HSPC) potentially causing leukemic transformation. Although the source of bystander signals is evident, the identification and characterization of these signals is challenging. Here, RIBE were analyzed in human CD34+ cells cultured in distinct molecular size fractions of medium conditioned by 2 Gy irradiated human MSC. Specifically, $\gamma \mathrm{H} 2 \mathrm{AX}$ foci (as a marker of DNA double-strand breaks) and chromosomal instability were evaluated in CD34+ cells grown in approximate (I) $<10 \mathrm{kDa}$, (II) $10-100 \mathrm{kDa}$ and (III) $>100 \mathrm{kDa}$ fractions of MSC conditioned medium and un-/fractionated control medium, respectively. Hitherto, significantly increased numbers of $\gamma \mathrm{H} 2 \mathrm{AX}$ foci and aberrant metaphases were detected in CD34+ cells grown in the (II) 10-100 kDa fraction when compared to (I) $<10 \mathrm{kDa}$ or (III) $>100 \mathrm{kDa}$ fractions or un-/fractionated control medium. Furthermore, RIBE disappeared after heat inactivation of medium at $75{ }^{\circ} \mathrm{C}$. Taken together, our data suggest that RIBE are mainly mediated by the heat-sensitive (II) 10-100 kDa fraction of MSC conditioned medium. We postulate proteins as RIBE mediators and in-depth proteome analyses to identify key bystander signals, which is fundamental for the development of next-generation anti-leukemic drugs.
\end{abstract}

Keywords: irradiation; mesenchymal stromal cells; CD34+ cells; bystander signals; bystander effects; leukemia 


\section{Introduction}

Genotoxic bystander signals released from irradiated human mesenchymal stromal cells (MSC) may induce radiation-induced bystander effects (RIBE) in non-irradiated human hematopoietic stem and progenitor cells (HSPC) potentially initiating myeloid neoplasms (MN). In the 2016 WHO classification, $\mathrm{MN}$ that arise after irradiation therapy are referred to as therapy-related $\mathrm{MN}(\mathrm{t}-\mathrm{MN})$ [1]. As t-MN are characterized by high-risk genetic alterations [2,3] and a particularly worse prognosis [4,5], novel therapeutic strategies are urgently needed.

Generally, RIBE describe 'out-of-field' effects of irradiation in non-irradiated cells that are comparable to effects in irradiated cells. RIBE may emerge as DNA damage (e.g., increased $\gamma \mathrm{H} 2 \mathrm{AX}$ foci, gene mutations, chromosomal aberrations, micronuclei), cell death (e.g., apoptosis, necrosis) and induction of cell survival mechanisms (e.g., adaptive response, DNA repair) [6-9]. Bystander signals are assumed to be initiated in irradiated cells by calcium fluxes [10] and mitochondrial metabolites [11-13]. Then, small molecules like nitric oxide (NO) [14], reactive oxygen species (ROS) [15], nuclear factor-kappa B (NF-kappa B) [13], and transforming growth factor beta-1 (TGFbeta-1) [16,17] may pass through cell membranes and gap junctions from the intracellular towards the extracellular space $[18,19]$. Hereupon, the bystander signals might be transmitted to non-irradiated cells that are referred to as bystander cells. Finally, ROS generated by NADH oxidases [20] and distinct RIBE mediators may be induced in affected bystander cells, thereby potentially initiating malignant transformation.

The analysis of bystander signals is a cutting-edge field in leukemia research. Here, irradiated healthy human MSC and healthy human CD34+ cells from the same donors were investigated in an in vitro model system that enables characterization of genotoxic signaling factors. Specifically, molecular size fractions of MSC conditioned medium of approximate (I) $<10 \mathrm{kDa}$, (II) $10-100 \mathrm{kDa}$ and (III) $>100 \mathrm{kDa}$ molecular weight were used for culture of CD34+ cells of the same donors. Afterwards, RIBE were analyzed in exposed CD34+ cells in terms of DNA damage and chromosomal instability (CIN). The data may provide important information on the fraction of interest in MSC conditioned medium to be analyzed most profitable by in-depth proteome analysis for the identification of key bystander signals, which might contribute to the development of nextgeneration anti-leukemic drugs.

\section{Experiments}

\subsection{Preparation of Human Femoral Heads}

This study was approved by the Ethics Committee II, Medical Faculty Mannheim, Heidelberg University (no. 2019-1128N). Procedures were performed in accordance with the local ethical standards and the principles of the 1964 Helsinki Declaration and its later amendments. Written informed consent was obtained from all study participants. Femoral heads were collected from 6 patients with coxarthrosis ( 1 female, 5 males, mean age: 68 years) undergoing hip replacement.

\subsection{Isolation of Human MSC}

Bones were broken into fragments and incubated for $1 \mathrm{~h}$ at $37^{\circ} \mathrm{C}$ in phosphate-buffered saline (PBS) supplemented with $1 \mathrm{mg} / \mathrm{mL}$ collagenase type I (Thermo Fisher, Waltham, US). Supernatants were filtered in a cell strainer with $100 \mu \mathrm{m}$ nylon mesh pores (Greiner Bio-One, Kremsmünster, Austria). Afterwards, bone fragments retained in the cell strainer were transferred into StemMACS MSC Expansion Media XF (Miltenyi Biotec, Bergisch Gladbach, Germany) supplemented with 1\% penicillin/streptomycin. Then, adherent MSC were expanded in T175 flasks in a humidified $5 \% \mathrm{CO}_{2}$ atmosphere at $37^{\circ} \mathrm{C}$ and passaged at $80 \%$ confluency.

\subsection{Isolation of Human CD34+ Cells}

CD34+ cells were isolated from bone marrow mononuclear cells by Ficoll density gradient centrifugation and magnetic-activated cell sorting using CD34 antibody-conjugated microbeads (Miltenyi Biotec). CD34+ cells were grown in StemSpan SFEM II medium (Stemcell Technologies, 
Vancouver, Canada) supplemented with StemSpan Myeloid Expansion supplement (SCF, TPO, GCSF, GM-CSF) (Stemcell Technologies) and 1\% penicillin/streptomycin in a humidified $5 \% \mathrm{CO}_{2}$ atmosphere at $37^{\circ} \mathrm{C}$.

\subsection{Preparation of Fractions of MSC Conditioned Medium}

MSC were grown in T175 flasks until reaching $80 \%$ confluency. MSC were rinsed in PBS and fresh StemSpan SFEM II medium was added. Afterwards, MSC were 2 Gy irradiated by 6 MV x-rays in a Versa HD linear accelerator (Elekta, Stockholm, Sweden), while control MSC were not irradiated. MSC conditioned medium and control medium were obtained from irradiated and non-irradiated MSC, respectively, after $4 \mathrm{~h}$ incubation at $37^{\circ} \mathrm{C}$. The collected medium was centrifuged $(1.200 \mathrm{rpm}$, $10 \mathrm{~min}$ ) and supernatants were filtered through $10 \mathrm{kDa}$ molecular weight cut-off (MWCO) ultrafiltration centrifugal filter units (Amicon Ultra, Merck, Darmstadt, Germany) to obtain (I) approximate $<10 \mathrm{kDa}$ fractions of MSC conditioned and control medium, respectively. Next, the supernatant above the filter was adjusted with fresh medium to the original volume and filtered through $100 \mathrm{kDa}$ MWCO ultrafiltration centrifugal filter units to obtain (II) approximate 10-100 kDa fractions of MSC conditioned and control medium, respectively. Finally, the supernatant above the filter was adjusted with fresh medium to the original volume and then contained (III) approximate > $100 \mathrm{kDa}$ fractions of MSC conditioned and control medium, respectively. The distinct fractions (I)(III) of MSC conditioned and control medium were stored at $-20^{\circ} \mathrm{C}$.

\subsection{Heat Inactivation of MSC Conditioned and Control Medium}

Heat inactivation of RIBE mediators in un-/fractionated MSC conditioned medium and un-/ fractionated control medium was performed by incubation at $75{ }^{\circ} \mathrm{C}$ for $20 \mathrm{~min}$.

\subsection{RIBE Analysis}

RIBE were analyzed in CD34+ cell samples (6 patients) at day 6 after culture for 3 days in native medium followed by culture for 3 days in un-/fractionated MSC conditioned medium or in un- $/$ fractionated control medium, respectively. In addition, experiments with CD34+ cell samples (2 patients) were performed with MSC conditioned medium after heat inactivation.

\subsection{Immunofluorescence Staining of $\gamma H 2 A X$}

Immunofluorescence staining of $\gamma \mathrm{H} 2 \mathrm{AX}$ was performed in CD34+ cells using a JBW301 mouse monoclonal anti- $\gamma \mathrm{H} 2 \mathrm{AX}$ antibody (Merck) and an Alexa Fluor 488-conjugated goat anti-mouse secondary antibody (Thermo Fisher) [21,22]. At least 50 nuclei were analyzed in each sample.

\subsection{Cytogenetic Analysis}

Cytogenetic analysis of G-banded chromosomes was performed in CD34+ cells according to standard procedures [23]. At least 25 metaphases were analyzed in each sample following the ISCN 2016 [24]. Sporadic chromosomal alterations (e.g., chromatid breaks (chtb), chromosome breaks, trisomy) were included in the karyotype (non-clonal events) when detected in at least one metaphase. Because tetraploid/octaploid metaphases were detected at low frequency in CD34+ cells grown in control medium as well, they were only included in karyotypes in case of clonality (tetraploidy and/or octaploidy in two or more metaphases) according to the ISCN 2016.

\subsection{Statistical Analysis}

Statistical analysis was performed with SAS software, release 9.4 (SAS Institute, Cary, US). For quantitative variables, mean values and standard deviations were calculated. Categorical factors are presented with absolute and relative frequencies. In order to compare more than two groups, Kruskal-Wallis tests were performed. For pairwise group comparisons, exact Wilcoxon two-sample tests were used. In general, test results with $p<0.05$ was considered as statistically significant. 


\section{Results}

\subsection{Evaluation of Cell-Free MSC Conditioned Medium}

In order to prevent a transfer of MSC by MSC conditioned medium to the CD34+ cell cultures only centrifuged supernatants were used. In addition, (i) microscopic evaluation of supernatants in a Neubauer counting chamber, (ii) sterile filtration of supernatants and (iii) cytogenetic cross-over experiments using sexually divergent CD34+ cells and MSC could exclude transfer of MSC to the CD34+ cell cultures in our experiments.

\subsection{DNA Damage in Human CD34+ Cells}

$\gamma \mathrm{H} 2 \mathrm{AX}$ foci were analyzed in human CD34+ cell samples (4 patients; $\sum 32$ samples) expanded for 3 days in native medium followed by culture for 3 days in un-/fractionated MSC conditioned or un-/fractionated control medium, respectively (Figure 1a). Increased numbers of $\gamma \mathrm{H} 2 \mathrm{AX}$ foci (general $p=0.0068$ [Kruskal-Wallis test]; pairwise comparison each $p=0.0286$ [Wilcoxon two-sample test]) were detected in CD34+ cells grown in the (II) 10-100 kDa fraction of MSC conditioned medium (0.67 $\pm 0.10 \gamma \mathrm{H} 2 \mathrm{AX}$ foci per CD34+ cell; mean \pm standard error of mean) when compared to numbers of $\gamma \mathrm{H} 2 \mathrm{AX}$ foci in CD34+ cells grown in (I) $<10 \mathrm{kDa}(0.19 \pm 0.01 \gamma \mathrm{H} 2 \mathrm{AX}$ foci per CD34+ cell) and (III) $>$ $100 \mathrm{kDa}(0.23 \pm 0.04 \gamma \mathrm{H} 2 \mathrm{AX}$ foci per CD34+ cell) fractions or in un-/fractionated control medium $(0.12$ $\pm 0.01 \gamma \mathrm{H} 2 \mathrm{AX}$ foci per CD34+ cell). Since $\gamma \mathrm{H} 2 \mathrm{AX}$ foci are a marker of DNA double-strand breaks (DSB), our findings suggest that DNA damage signaling factors mainly localize in the (II) $10-100 \mathrm{kDa}$ fraction of MSC conditioned medium.

\section{Tetraploidy}

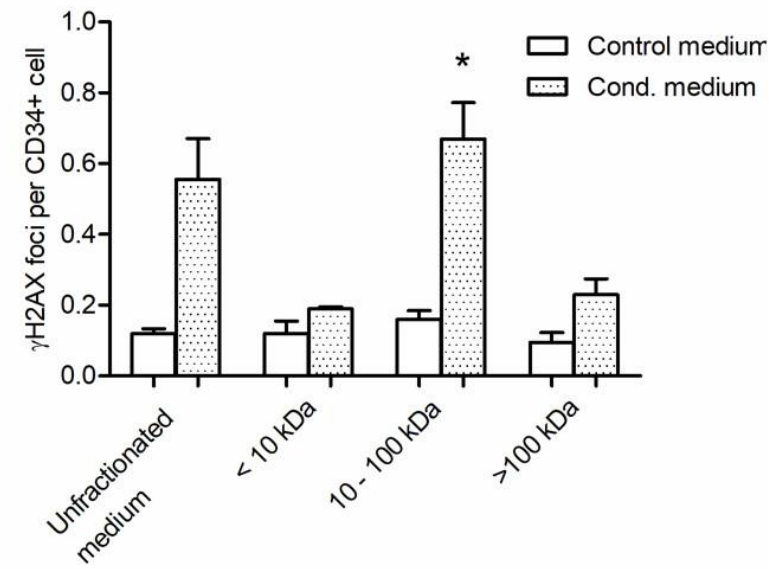

(a)

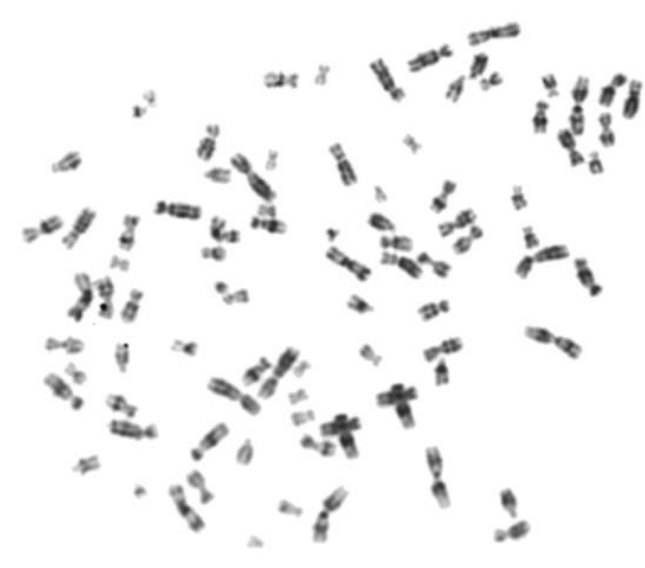

(b)

Figure 1. Radiation-induced bystander effects in CD34+ cells grown in distinct molecular size fractions of medium conditioned by 2 Gy irradiated mesenchymal stromal cells (MSC) and un-/ fractionated control medium. (a) $\gamma \mathrm{H} 2 \mathrm{AX}$ foci levels in CD34+ cells grown in (I) $<10 \mathrm{kDa}$, (II) $10-100$ $\mathrm{kDa}$ and (III) $>100 \mathrm{kDa}$ fractions of MSC conditioned medium and in un-/fractionated control medium. ${ }^{*} p=0.0068$ [Kruskal-Wallis test] and $p=0.0286$ [Wilcoxon two-sample test] when compared to numbers of $\gamma \mathrm{H} 2 \mathrm{AX}$ foci in CD34+ cells grown in (I) $<10 \mathrm{kDa}$ and (III) $>100 \mathrm{kDa}$ fractions or in un/fractionated control medium. (b) Exemplary tetraploidy of a CD34+ cell grown in the (II) 10-100 kDa fraction of MSC conditioned medium. 
Table 1. Radiation-induced bystander effects in CD34+ cells grown in un-/fractionated medium conditioned by 2 Gy irradiated mesenchymal stromal cells.

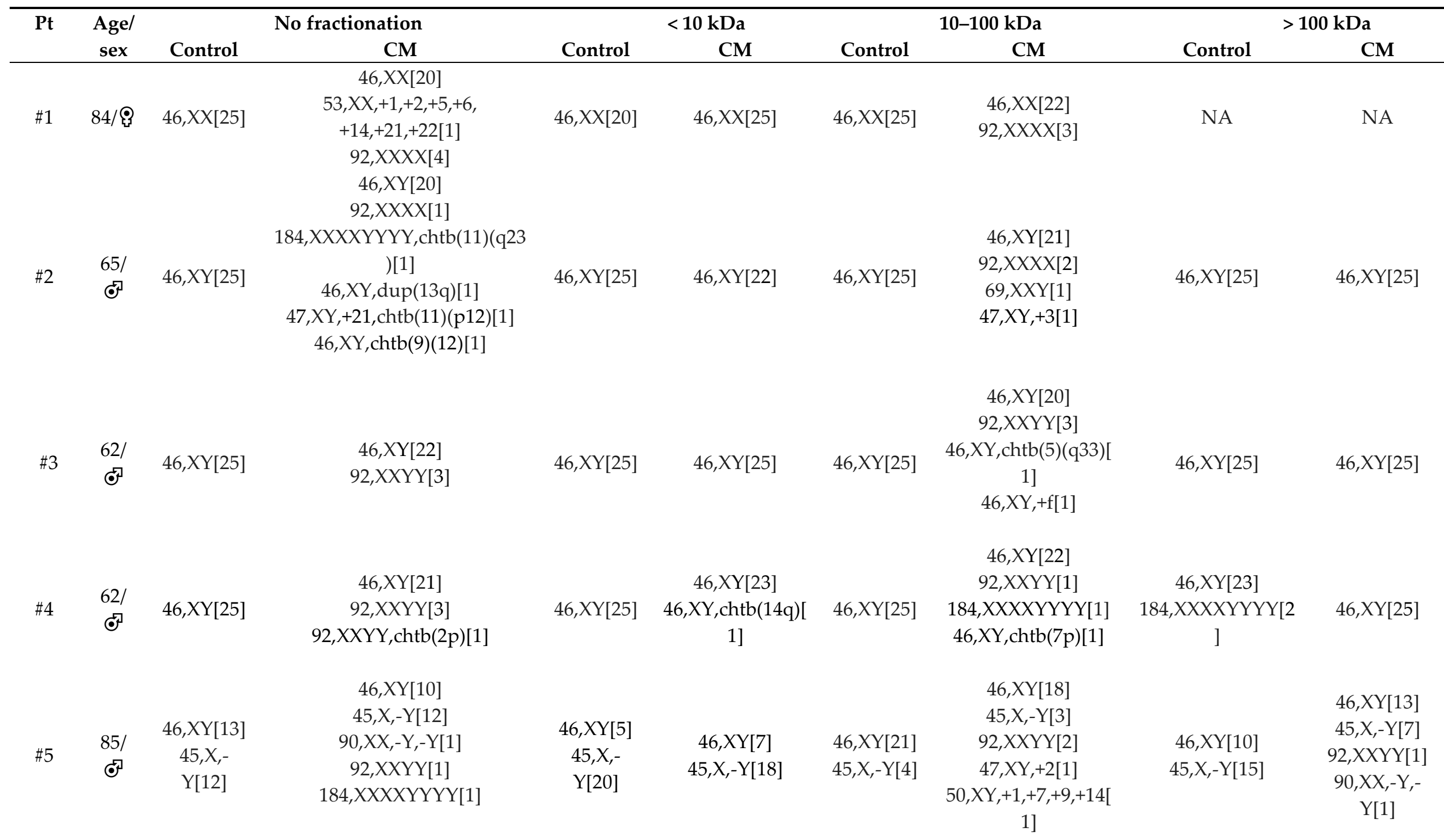




\begin{tabular}{|c|c|c|c|c|c|c|c|c|c|}
\hline & & & $46, X Y[22]$ & & & & 46,XY[21] & & \\
\hline
\end{tabular}

$\mathrm{CM}$, conditioned medium; NA, not assessed. 


\subsection{Chromosomal Instability in Human CD34+ Cells}

Metaphases were analyzed in human CD34+ cell samples (6 patients; $\sum 46$ samples) expanded for 3 days in native medium followed by culture for 3 days in un-/fractionated MSC conditioned or un-/fractionated control medium, respectively (Figure 1b, Table 1). Increased numbers of aberrant metaphases (general $p=0.0007$ [Kruskal-Wallis test]; pairwise comparison each $p=0.0022$ [Wilcoxon two-sample test]) were detected in CD34+ cells grown in the (I) 10-100 kDa fraction of MSC conditioned medium when compared to numbers of aberrant metaphases in CD34+ cells grown in (II) $<10 \mathrm{kDa}$ and (III) $>100 \mathrm{kDa}$ fractions of MSC conditioned medium or in un-/fractionated control medium. More precisely, distinct chromatid breaks (chtb), e.g., chtb(5q) and $\operatorname{chtb}(7 q)$ as well as aneuploidies, e.g., tetraploidies and octaploidies, were observed in CD34+ cells grown in the (II) 10$100 \mathrm{kDa}$ fraction of MSC conditioned medium. In addition, distinct chtb, e.g., chtb(2), chtb(9) and chtb(11) as well as aneuploidies, e.g., tetraploidies and octaploidies, were observed in CD34+ cells grown in unfractionated MSC conditioned medium. It has to be noted, that loss of chromosome $\mathrm{Y}$ in \#5 is a common finding in elderly men occurring at a frequency of $5-10 \%[25,26]$. Further, few chromosomal aberrations, e.g., chtb(14q) and aneuploidies, e.g., tetraploidies, were detected at very low frequencies in (I) $<10 \mathrm{kDa}$ and (III) $>100 \mathrm{kDa}$ fractions of MSC conditioned medium, which might be due to limitations in accuracy of the filtration process.

Finally, heat inactivation of unfractionated MSC conditioned medium and unfractionated control medium (2 patients, $\sum 4$ samples) resulted in reduced proliferation of CD34+ cells. Here, all evaluable metaphases displayed a normal karyotype.

\section{Discussion}

Genotoxic bystander signals released from irradiated human MSC may induce DNA damage and CIN in human HSPC potentially initiating MN. While increased DNA damage and CIN are readily inducible in human CD34+ cells by exposure to MSC conditioned medium, the genotoxic bystander signals in MSC conditioned medium remain largely uncharacterized yet. Therefore, our study was designed to investigate the molecular features of bystander signals in terms of molecular weight and potential protein characteristics. For this purpose, approximate (I) $<10 \mathrm{kDa}$, (II) $10-100$ $\mathrm{kDa}$ and (III) $>100 \mathrm{kDa}$ fractions of MSC conditioned medium were generated for co-culture experiments in healthy human CD34+ cells of the same donors.

Increased numbers of $\gamma \mathrm{H} 2 \mathrm{AX}$ foci were detected in CD34+ cells grown in the (II) $10-100 \mathrm{kDa}$ fraction of MSC conditioned medium when compared to low numbers of $\gamma \mathrm{H} 2 \mathrm{AX}$ foci in CD34+ cells grown in (I) $<10 \mathrm{kDa}$ and (III) $>100 \mathrm{kDa}$ fractions of MSC conditioned medium or in un-/fractionated control medium. As $\gamma \mathrm{H} 2 \mathrm{AX}$ foci are a marker of DSB, our data are in line with similarly increased numbers of chtb detected in CD34+ cells grown in the (II) 10-100 kDa fraction of MSC conditioned medium. Importantly, chtb may activate oncogenes or inactivate tumor suppressor genes, respectively, thus providing a potential mechanistic link to the initiation of MN [27].

Further, increased numbers of aberrant metaphases were observed in CD34+ cells grown in the (II) 10-100 kDa fraction of MSC conditioned medium when compared to low numbers of aberrant metaphases in CD34+ cells grown in (I) $<10 \mathrm{kDa}$ and (III) $>100 \mathrm{kDa}$ fractions of MSC conditioned medium or in un-/fractionated control medium. In particular, the number of tetraploidies was increased in the (II) 10-100 kDa fraction of MSC conditioned medium. Generally, tetraploidies may occur by chromosomal non-disjunction during mitosis or cytokinesis failure [28]. Further, tetrapolidies are found in about $1 \%$ of AML but $13 \%$ of $t$-AML cases [29]. Hence, our finding of increased tetraploidies in CD34+ cells grown in the (II) 10-100 kDa fraction of MSC conditioned medium suggests a mechanistic link to the initiation of MN. Although tetraploidies occurred at very low frequency in CD34+ cells grown in control medium, this result is not contradictory to our interpretations but indicates that tetraploidies may randomly occur in vitro during the proliferation process itself.

Finally, heat inactivation of unfractionated MSC conditioned and control medium resulted in reduced proliferation of CD34+ cells, which all demonstrated regular karyotypes. Thus, RIBE 
mediators have a temperature-sensitive structure, supporting the notion that the three-dimensional conformation of macromolecules, such as the native tertiary structure in proteins, confers specifically to the genotoxic effects in the (II) $10-100 \mathrm{kDa}$ fraction of MSC conditioned medium instead of the sheer presence of mediating macromolecules.

Our study may raise the question for the impact of ROS and NO as potential RIBE mediators in the 10-100 kDa fraction of MSC conditioned medium. Considering that ROS and NO are rather shortlived mediator molecules, there might be no major impact of MSC released ROS and NO on detected RIBE in CD34+ cells in our experiments. More likely, hitherto unknown mediators with a longer halflife may increase ROS and NO in exposed CD34+ cells grown in MSC conditioned medium [20].

\section{Conclusions}

In conclusion, our data demonstrate that substantial genotoxic bystander signals mainly localize in the (II) 10-100 kDa fraction of MSC conditioned medium and that these signals are heat-sensitive. Based on these biochemical properties, we postulate proteins as RIBE mediators, which should be further analyzed by an in-depth proteome analysis of the corresponding fraction. Ultimately, it has the potential to uncover the identity of key bystander signals, which is fundamental for the development of next-generation anti-leukemic drugs.

Author Contributions: Conceptualization, H.D.P. and O.D.; methodology, H.D.P and O.D.; software, V.K. and A.F.; validation, V.K., A.F. and H.D.P.; formal analysis, C.W.; investigation, V.K., S.B. and A.F.; resources, H.K., A.D., A.J., M.B., W.S., A.F. and W.-K.H.; data curation, V.K., A.F. and H.D.P.; writing-original draft preparation, H.D.P; writing-review and editing, H.D.P., O.D., J.F., W.S., A.F. and W.-K.H.; visualization, H.D.P.; supervision, A.F. and W.-K.H.; project administration, H.D.P.; funding acquisition, H.D.P. and A.F. All authors have read and agreed to the published version of the manuscript.

Acknowledgments: This research was funded by Deutsche José Carreras Leukämie-Stiftung, DJCLS 14 R/2017.

Conflicts of Interest: The authors declare no conflict of interest.

\section{Abbreviations}

chtb: chromatid breaks

CIN: chromosomal instability

DSB: DNA double-strand breaks

HSPC: human hematopoietic stem and progenitor cells

MN: myeloid neoplasms

MSC: mesenchymal stromal cells

NO: nitric oxide

PBS: phosphate-buffered saline

RIBE: radiation-induced bystander effects

ROS: reactive oxygen species

t-MN: therapy-related $\mathrm{MN}$

\section{References}

1. Vardiman: J.W.; Arber, D.A.; Brunning, R.D., Larson, R.A., Matutes, E., Baumann, I., Kvasnicka, H.M. Therapy-related myeloid neoplasms. In WHO Classification of Tumours of Haematopoietic and Lymphoid Tissues, 4th ed.; Swerdlow, S.H., Campo, E., Harris, N.L., Jaffe E.S., Pileri, S.A., Stein, H., Thiele, J., Arber, D.A., Hasserjian, R.P., Le Beau, M.M., Orazi, A., Siebert, R., Eds.; International Agency for Research on Cancer: Lyon, France, 2017; pp. 153-155.

2. Ok, C.Y.; Patel, K.P.; Garcia-Manero, G.; Routbort, M.J.; Fu, B.; Tang, G.; Goswami, M.; Singh, R.; KanagalShamanna, R.; Pierce, S.A.; et al. Mutational profiling of therapy-related myelodysplastic syndromes and acute myeloid leukemia by next generation sequencing, a comparison with de novo diseases. Leuk Res. 2015, 39, 348-354.

3. Wong, T.N.; Ramsingh, G.; Young, A.L.; Miller, C.A.; Touma, W.; Welch, J.S.; Lamprecht, T.L.; Shen, D.; Hundal, J.; Fulton, R.S.; et al. Role of TP53 mutations in the origin and evolution of therapy-related acute myeloid leukaemia. Nature 2015, 518, 552-555. 
4. Fianchi, L.; Pagano, L.; Piciocchi, A.; Candoni, A.; Gaidano, G.; Breccia, M.; Criscuolo, M.; Specchia, G.; Maria Pogliani, E.; Maurillo, L.; et al. Characteristics and outcome of therapy-related myeloid neoplasms: Report from the Italian network on secondary leukemias. Am. J. Hematol. 2015, 90, E80-85.

5. Granfeldt Ostgard, L.S.; Medeiros, B.C.; Sengelov, H.; Norgaard, M.; Andersen, M.K.; Dufva, I.H.; Friis, L.S.; Kjeldsen, E.; Marcher, C.W.; Preiss, B.; et al. Epidemiology and Clinical Significance of Secondary and Therapy-Related Acute Myeloid Leukemia: A National Population-Based Cohort Study. J. Clin. Oncol. 2015, 33, 3641-3649.

6. Azzam, E.I.; de Toledo, S.M.; Raaphorst, G.P.; Mitchel, R.E., Low-dose ionizing radiation decreases the frequency of neoplastic transformation to a level below the spontaneous rate in C3H 10T1/2 cells. Radiat. Res. 1996, 146, 369-373.

7. Watson, G.E.; Lorimore, S.A.; Macdonald, D.A.; Wright, E.G., Chromosomal instability in unirradiated cells induced in vivo by a bystander effect of ionizing radiation. Cancer Res. 2000, 60, 5608-5611.

8. Nagasawa, H.; Little, J.B., Induction of sister chromatid exchanges by extremely low doses of alphaparticles. Cancer Res. 1992, 52, 6394-6396.

9. Zhou, H.; Randers-Pehrson, G.; Waldren, C.A.; Vannais, D.; Hall, E.J.; Hei, T.K., Induction of a bystander mutagenic effect of alpha particles in mammalian cells. Proc. Natl. Acad Sci. U. S. A. 2000, 97, 2099-2104.

10. Shao, C.; Lyng, F.M.; Folkard, M.; Prise, K.M., Calcium fluxes modulate the radiation-induced bystander responses in targeted glioma and fibroblast cells. Radiat. Res. 2006, 166, 479-487.

11. Chen, S.; Zhao, Y.; Han, W.; Zhao, G.; Zhu, L.; Wang, J.; Bao, L.; Jiang, E.; Xu, A.; Hei, T.K.; et al. Mitochondria-dependent signalling pathway are involved in the early process of radiation-induced bystander effects. Br. J. Cancer 2008, 98, 1839-1844.

12. Tartier, L.; Gilchrist, S.; Burdak-Rothkamm, S.; Folkard, M.; Prise, K.M., Cytoplasmic irradiation induces mitochondrial-dependent 53BP1 protein relocalization in irradiated and bystander cells. Cancer Res. 2007, $67,5872-5879$.

13. Zhou, H.; Ivanov, V.N.; Lien, Y.C.; Davidson, M.; Hei, T.K., Mitochondrial function and nuclear factorkappaB-mediated signaling in radiation-induced bystander effects. Cancer Res. 2008, 68, 2233-2240.

14. Shao, C.; Stewart, V.; Folkard, M.; Michael, B.D.; Prise, K.M., Nitric oxide-mediated signaling in the bystander response of individually targeted glioma cells. Cancer Res. 2003, 63, 8437-8442.

15. Wang, R.; Coderre, J.A., A bystander effect in alpha-particle irradiations of human prostate tumor cells. Radiat. Res. 2005, 164, 711-722.

16. Shao, C.; Folkard, M.; Prise, K.M., Role of TGF-beta1 and nitric oxide in the bystander response of irradiated glioma cells. Oncogene 2008, 27, 434-440.

17. Gow, M.D.; Seymour, C.B.; Ryan, L.A.; Mothersill, C.E., Induction of bystander response in human glioma cells using high-energy electrons: A role for TGF-beta1. Radiat. Res. 2010, 173, 769-778.

18. Shao, C.; Furusawa, Y.; Aoki, M.; Ando, K., Role of gap junctional intercellular communication in radiationinduced bystander effects in human fibroblasts. Radiat. Res. 2003, 160, 318-323.

19. Azzam, E.I.; de Toledo, S.M.; Little, J.B., Direct evidence for the participation of gap junction-mediated intercellular communication in the transmission of damage signals from alpha -particle irradiated to nonirradiated cells. Proc. Natl. Acad Sci. U. S. A. 2001, 98, 473-478.

20. Thannickal, V.J.; Fanburg, B.L., Activation of an H2O2-generating NADH oxidase in human lung fibroblasts by transforming growth factor beta 1. J. Biol. Chem. 1995, 270, 30334-30338.

21. Popp, H.D.; Naumann, N.; Brendel, S.; Henzler, T.; Weiss, C.; Hofmann, W.K.; Fabarius, A., Increase of DNA damage and alteration of the DNA damage response in myelodysplastic syndromes and acute myeloid leukemias. Leuk Res, 2017, 57, 112-118.

22. Popp, H.D.; Brendel, S.; Hofmann, W.K.; Fabarius, A. Immunofluorescence Microscopy of gammaH2AX and 53BP1 for Analyzing the Formation and Repair of DNA Double-strand Breaks. J. Vis. Exp. 2017, e56617.

23. Löffler, H.; Rastetter, J.; Haferlach, T. Light microscopic procedures. In Atlas of Clinical Hematology, 6th ed.; Springer: Heidelberg, Germany, 2005; p. 8.

24. McGowan-Jordan, J., Simons, A., Schmid, M., ISCN 2016 An International System for Human Cytogenetic Nomenclature (2016). Karger: Basel, 2016.

25. Jacobs, P.A.; Brunton, M.; Court Brown, W.M.; Doll, R.; Goldstein, H., Change of human chromosome count distribution with age: Evidence for a sex differences. Nature 1963, 197, 1080-1081.

26. Pierre, R.V.; Hoagland, H.C., Age-associated aneuploidy: Loss of Y chromosome from human bone marrow cells with aging. Cancer 1972, 30, 889-894. 
27. American Cancer Society. Oncogenes and tumor suppressor genes. Available online: https://www.cancer.org/cancer/cancer-causes/genetics/genes-and-cancer/oncogenes-tumor-suppressorgenes.html (accessed on 25 October 2020).

28. Tanaka, K.; Goto, H.; Nishimura, Y.; Kasahara, K.; Mizoguchi, A.; Inagaki, M., Tetraploidy in cancer and its possible link to aging. Cancer Sci 2018, 109, 2632-2640.

29. Huang, L.; Wang, S.A.; DiNardo, C.; Li, S.; Hu, S.; Xu, J.; Zhou, W.; Goswami, M.; Medeiros, L.J.; Tang, G., Tetraploidy/near-tetraploidy acute myeloid leukemia. Leuk Res 2017, 53, 20-27.

Publisher's Note: MDPI stays neutral with regard to jurisdictional claims in published maps and institutional affiliations.

(C) 2020 by the authors; licensee MDPI, Basel, Switzerland. This article is an open access article distributed under the terms and conditions of the Creative Commons by Attribution (CC-BY) license (http://creativecommons.org/licenses/by/4.0/). 